\title{
An unusual presentation of atrial fibrillation
}

\author{
Mohammad Haqqi MD¹, Adrian Baranchuk MD FACC ${ }^{1}$, Alexander Boag MD², Glorianne Ropchan MD FRCSC ${ }^{3}$
}

A 66-year-old man with a history of smoking and chronic obstructive pulmonary disease presented to the emergency department with a two-month history of worsening shortness of breath. His electrocardiogram revealed new-onset atrial fibrillation. Adequate rate control was achieved with beta-blockers and digoxin (Figure 1A).

A chest $\mathrm{x}$-ray was compatible with chronic obstructive pulmonary disease. A subsequent echocardiogram revealed a mass filling the left atrium and obstructing the mitral orifice (Figure 1B). A cine magnetic resonance imaging sequence showed a pedunculated mass arising from the mid portion of the interatrial septum and measuring $7.8 \mathrm{~cm} \times$ $4.8 \mathrm{~cm}$ in the maximum transverse anteroposterior dimension (Figure 1C). It occupied the entire left atrial cavity and protruded across the mitral valve annulus into the base of the left ventricular (LV) cavity during systole. A coronary angiogram was normal. The patient was taken to the operating room for surgical removal of a large left atrial mass with excision of the site of septal attachment. Bovine pericardial patch repair of the iatrogenic atrial septal defect was performed.

Macroscopically (Figure 1D), the mass consisted of white, soft, oblong tissue weighing $109 \mathrm{~g}$. The cut surface of the mass was yellow and glistening, with focal areas of hemorrhage and necrosis. Microscopic pathology (Figure 1E) showed typical histological features of a cardiac myxoma with no atypia or malignancy.

Primary tumours of the heart are extremely rare. Cardiac myxomas account for $50 \%$ of all primary cardiac tumours. They are more common in the female population, and typically occur between 30 and 60 years of age. Most myxomas originate from the interatrial septum at the fossa ovalis. Myxomas can present with obstructive and constitutional symptoms, electrical disturbances, and emboli. Surgical excision
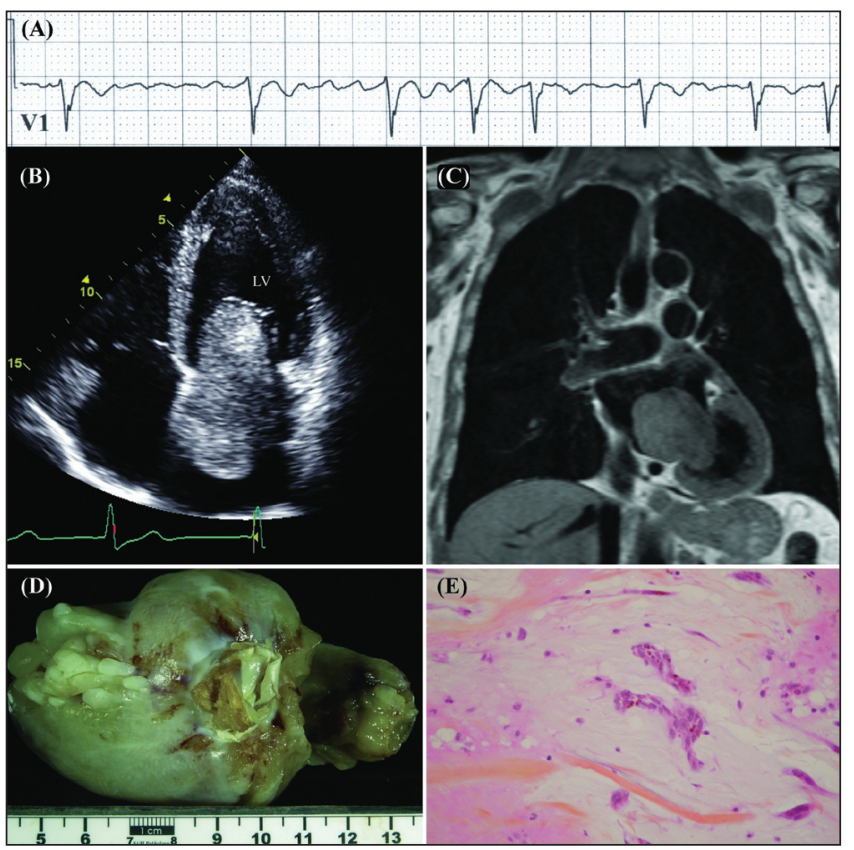

Figure 1

is usually curative. The present patient was discharged home in normal sinus rhythm six days after surgery.

${ }^{1}$ Cardiology Division; ${ }^{2}$ Department of Pathology and Molecular Medicine; ${ }^{3}$ Cardiac Surgery Division, Kingston General Hospital, Queen's University,

Kingston, Ontario

Correspondence: Dr Adrian Baranchuk, Cardiology Division, Kingston General Hospital, Queen's University, FAPC 3, 76 Stuart Street, Kingston,

Ontario K7L 2V7. Telephone 613-549-6666 ext 3801, fax 613-548-1387, e-mail barancha@kgh.kari.net

Received for publication April 23, 2009. Accepted May 17, 2009 